\title{
Is Serotonin the Missing Link between COVID-19 Course of Severity in Patients with Diabetes and Obesity?
}

\author{
Ana Paula Santos ${ }^{a, b}, c$ Carlos Ferreira Couto ${ }^{d}$ Sofia Silva Pereira ${ }^{e, f}$ \\ Mariana Pereira Monteiro ${ }^{e} f$ \\ aPortuguese Oncology Institute of Porto (IPO Porto), Department of Endocrinology, Porto, Portugal; bPortuguese \\ Oncology Institute of Porto (IPO Porto), Clinical Research Unit, Research Center of IPO Porto (CI-IPOP), Porto, \\ Portugal; 'Precancerous and Early Cancer Management Group, Research Center of IPO Porto (CI-IPOP), \\ RISE@CI-IPOP (Health Research Network), Porto, Portugal; 'Lisbon University Institute (ISCTE-IUL), Lisbon, Portugal; \\ 'Clinical and Experimental Endocrinology, Unit for Multidisciplinary Research in Biomedicine, UMIB ICBAS, \\ University of Porto, Porto, Portugal; fInstitute of Biomedical Sciences Abel Salazar (ICBAS), Department of Anatomy, \\ University of Porto, Porto, Portugal
}

\section{Keywords}

COVID-19 · Type 2 diabetes · Obesity · Serotonin

\begin{abstract}
COVID-19 is an intriguing infectious condition with multisystemic manifestations and variable outcomes that are influenced by the concomitant presence of non-communicable diseases, such as obesity, diabetes, and cardiovascular disease, which were previously well established epidemics and therefore are considered global syndemics. Although an enormous progress towards understanding mechanisms of SARS-CoV-2 infection leading to COVID-19 has been made, there are still many areas of uncertainty to clarify. Systemic diseases are characterized by common links that allow integrating apparently unrelated disease manifestations. The authors launch the provocative hypothesis that serotonin is the putative mediator linking the lung, gut, cardiac, neurological, and other systemic manifestations that characterize severe COVID-19 in individuals with diabetes and obesity. In support of a role for serotonin in the mechanisms leading to disease severity are the similarities between acute and postacute COVID-19 manifestations and neuroendocrine tumors presenting with carcinoid syndrome. Scientific discussion is
\end{abstract}

set by highlighting the available clues that support this working hypothesis to trigger future research aimed at unravelling the molecular pathways underlying SARS-CoV-2 infection that are still far from being fully disclosed.

(c) 2022 S. Karger AG, Basel

\section{Introduction}

Interaction between immune and neuroendocrine systems throughout lung and gut epitheliums is crucial in the response to environmental aggressions, such as infectious agents and allergens, as well as in chronic inflammatory conditions [1]. Neuro-immune cell units are defined as immune and neuronal cells anatomically co-localized that functionally interact to steer tissue physiology and protection. Neuro-immune cell units are important orchestrators of multiple physiological processes including inflammation, tissue repair, and thermogenesis, among many others [2]. Cytokines liberated by immune cells and hormones, neurotransmitters secreted by neuroendocrine cells are key mediators between the two systems and responsible for acute and chronic responses to aggression.
Correspondence to:

Ana Paula Santos, anapaulasantos@ipoporto.min-saude.pt 


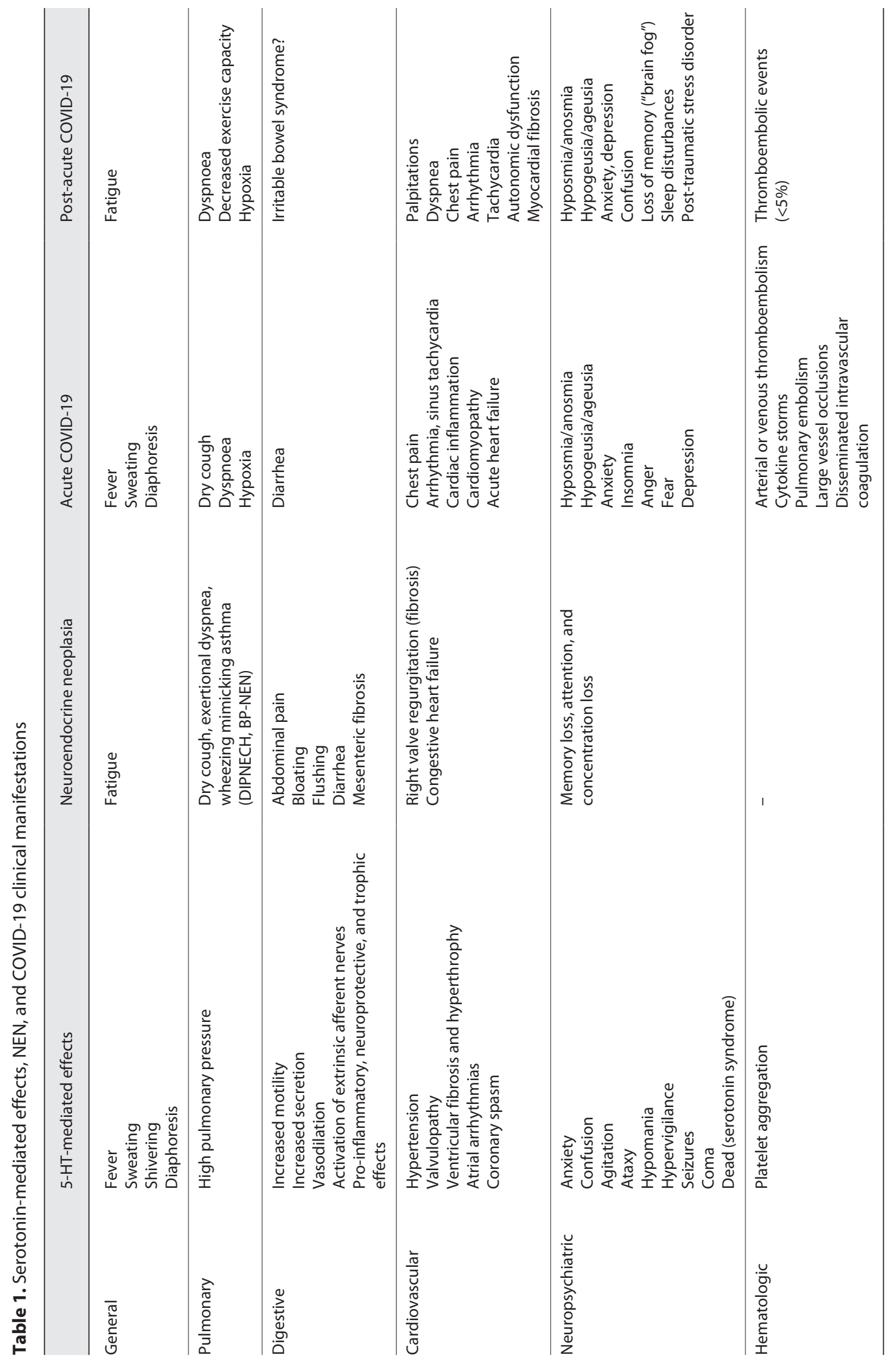




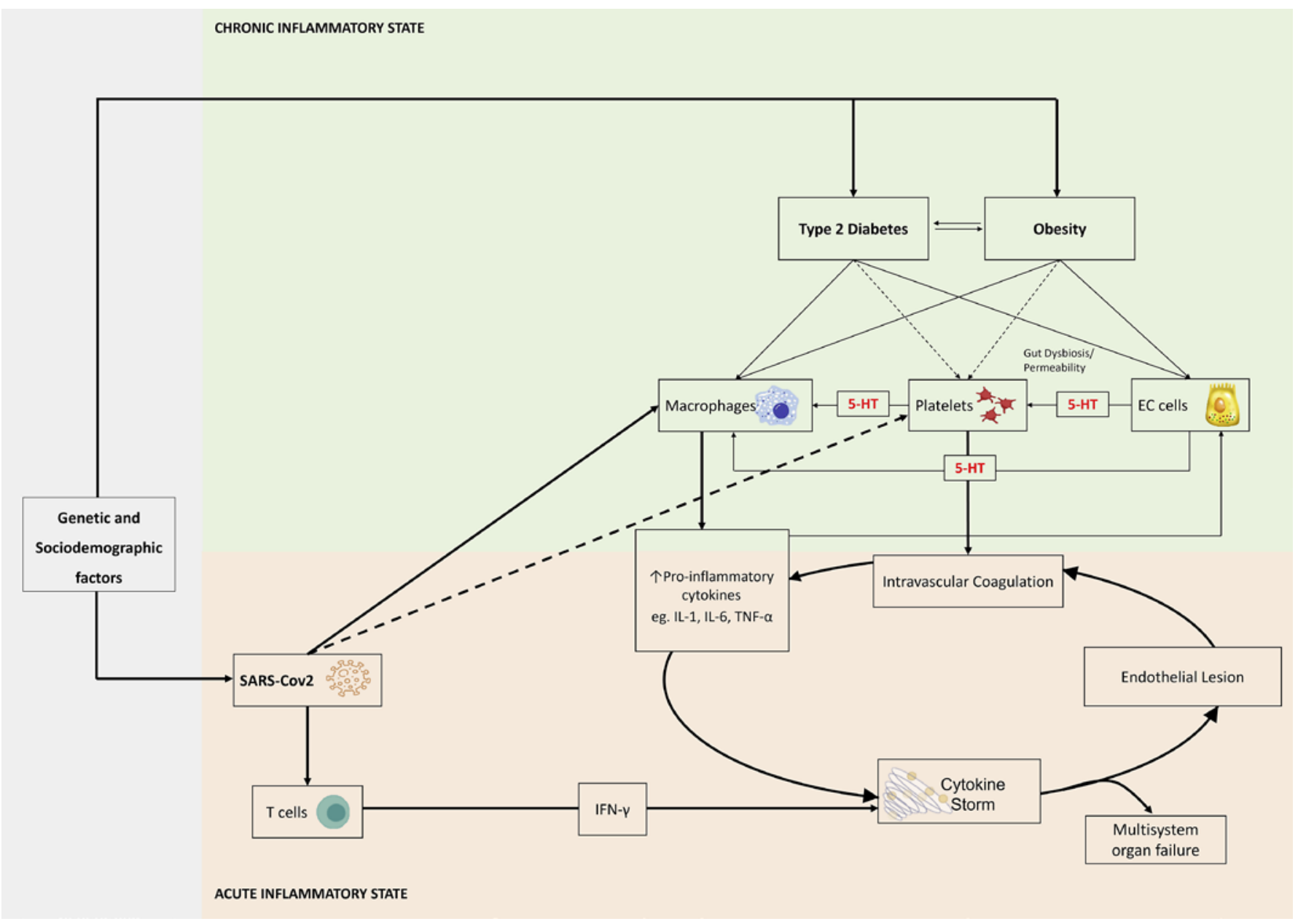

Fig. 1. Putative mechanisms linking obesity, T2D, and serotonin (5-HT), with SARS-CoV-2 infection severity.

\section{SARS-CoV-2 as a Systemic Inflammatory Disease in Obese and Diabetic Patients: Interplay with Neuropeptides in Neuroendocrine Neoplasia}

SARS-CoV-2 infection is consensually accepted as a multisystem acute disease involving several systems and organs, with particular emphasis on the lungs and gut, which in more severe cases can lead to well-documented cytokine and chemokine storm (Table 1) [3]. Diseases characterized by low grade chronic inflammatory states, such as obesity $[4,5]$, visceral obesity defined as a high waist-to-hip ratio [6], and type 2 diabetes (T2D) [7], provide a substantial increase in the risk of SARS-CoV-2 infection morbidity and mortality as shown in Figure 1 [8]. Adipose tissue is well documented to act as viral reservoir. Angiotensin-converting enzyme 2, which serves as receptor for SARS-CoV-2 to initiate infection, is widely ex- pressed in adipocytes, and its expression is enriched in adipocytes of individuals with obesity and T2D [9].

Insulin resistance (IR) is a state of subclinical metabolic endotoxemia characterized by a pro-inflammatory and prothrombotic state with overproduction of mediators including cytokines (interleukin 6 [IL-6], tumor necrosis factor, among others) and fibrinogen. Subsequent endothelial dysfunction and thrombosis are the ultimate mechanisms that lead to cardiovascular disease in obesity and T2D [10]. All those mechanisms involved in chronic inflammation states that characterize IR, obesity, T2D, and cardiovascular disease are acutely over activated in SARS-CoV-2 infection and seem to be responsible for the multisystemic manifestations of the disease and poorer patient outcomes, in subjects with those comorbid conditions.

IR along with compensatory hyperinsulinism is hypothesized to be one of the major mechanisms underlying 
the positive association of obesity and T2D with several malignancies $[11,12]$. Well differentiated gastro-enteropancreatic neuroendocrine neoplasia (NEN) was also recently described to be associated with visceral obesity and elevated fasting plasma glucose [13], while the available data suggests the putative role of inflammatory cytokines, such as interleukin-2 (IL-2) in functioning $[14,15]$ and IL-6 in both nonfunctioning pancreatic NEN and gastrointestinal NEN [14].

Serotonin (5-hydroxytryptamine or 5-HT) is a key neurotransmitter synthesized by specialized enterochromaffin cells within GI mucosa, so-called enteroendocrine cells (EC). The pulmonary endothelium is capable of removing and metabolizing 5 -HT carried by the venous blood, while there is evidence that impairment of uptake of 5-HT by the lung might portend more serious endothelial damage, whenever the barrier function of the endothelium is compromised [16]. 5 -HT is the main responsible for typical carcinoid syndrome manifestations, including secretory diarrhea and flushing that are common clinical features in patients with small intestinal NEN [17], as well as allergic reactions and bronchospasm causing atypical carcinoid syndrome, which are present in 1-5\% of functioning bronchopulmonary carcinoids [18]. 5-HT is also responsible for the mesenteric and cardiac valve fibrosis, associated with carcinoid-related advanced disease [19, 20]. Besides these well-documented 5-HT effects, other peripheral actions were recently described, namely a role in glucose homeostasis regulation, lipid metabolism, bone density, and diseases associated with metabolic syndrome, such as obesity and T2D. So, 5-HT seems to act as a key neurotransmitter that mediates the GI luminal sensory role of EC, as these cells can integrate clues from ingested nutrients, the enteric nervous system, and the gut microbiome [21]. Whether 5-HT is also involved in the mechanisms that could explain SARS-CoV-2 induced acute hyperglycemia state is unknown, but interestingly, 5-HT levels were found to be elevated in patients with COVID-19 and diarrhea, as well as positively correlated with IL-6 levels and disease severity [22, 23]. 5-HT and dopamine also seem to be involved in the neurological manifestations of the SARS-CoV-2 induced disease, as defective angiotensinconverting enzyme 2 expression might be paralleled by dopa decarboxylase dysfunction, leading to altered neurotransmitters' levels [24]. Besides, 5-HT is involved in human taste signaling and threshold [25] in both rodents and insects, since one of the main role is to indirectly suppress the inputs at the first stage of ol- factory processing [26]. Loss of taste and smell are frequent manifestations of COVID-19 infection [27]. Recently, thrombosis and hemorrhage were described as rare side effects of SARS-CoV-2 vaccines. This phenomenon is called "Vaccine Induced Immune Thrombotic Thrombocytopenia," and was demonstrated to be caused by antibodies antiplatelet factor 4, the same mechanism that underlies heparin-induced thrombocytopenia, a rare clotting disorder that affects $1-2 \%$ of individuals after heparin exposure [28]. 5-HT release assay that measures 5-HT release from dense granules when platelets are activated is used as a "gold standard" to confirm heparin-induced thrombocytopenia [29]. Interestingly, platelets granules store the largest pool of peripheral 5-HT at millimolar concentrations. Platelet activation leads to granules release and thereby increases local and systemic 5-HT levels. 5-HT levels not only contribute to regulate vascular tone, hemostasis, and clot formation but also have direct effects on immune functions [30]. The use of selective serotonin reuptake inhibitors, as antidepressants were associated with lower risk of intubation or even death in patients hospitalized for COVID-19 [31]. Patients with COVID-19 treated with the selective serotonin reuptake inhibitor fluvoxamine presented lower risk of hospitalization $[32,33]$. Also, serotonin receptor (5HT-2) antagonists seem to reverse serotonin-mediated pulmonary vasoconstriction, lessen pulmonary platelet trapping, inhibit platelet activation and aggregation, and normalize increased respiratory drive, which is common pulmonary findings in severe COVID-19 [34]. In addition, the use of 5-HT2A receptor antagonists in patients with severe COVID-19 was found to be inversely associated with mortality [35]. Overall, these studies point toward a role for serotonin in COVID-19, acting via 5-HT2A receptor and 5-HT transporter. Although other serotonergic system components may also be involved in the mechanisms of COVID-19, those known to be involved in immune modulation, more evidence is still needed.

Moreover, in support of a role for serotonin in the mechanisms leading to disease severity are the similarities between lung manifestations observed in COVID-19 and diffuse idiopathic pulmonary neuroendocrine cell hyperplasia [36, 37], as well as the fact that neuroendocrine tumors presenting with typical or atypical carcinoid syndrome, mediated by 5-HT among other neuropeptides, are recognized to affect predominantly the lung and gut [38]. In addition, gastro-entero-pancreatic NEN are often associated with visceral obesity and high fasting plasma glucose levels [13], while COVID-19 related cyto- 
kine storm tends to occur more frequently in patients with obesity and T2D, which also points towards common systemic inflammatory pathways and further suggests that these entities could potentially share the same neuroendocrine mediators.

The chronicity of COVID-19 is now stated as long COVID since a wide spectrum of long-term sequelae in postinfectious disease is being described [39, 40]. Dyspnea, decreased exercise capacity and hypoxia are common manifestations, which result from reduced oxygen diffusion capacity, restrictive respiratory function as well the presence of ground-glass opacities and fibrotic changes on lung computed tomography [40]. Late complications of SARS-CoV-2 lung disease also include interstitial pulmonary fibrosis that can occur in up to $17 \%$ of the patients [41]. The true mechanisms for this late complication are unknown but renin-angiotensin system and acute inflammatory mediators, such as interleukins (IL-6, IL-1), vascular epithelial growth factor, and TGF-beta, as well as the exacerbated wound healing response caused by chronic activation of fibrinogen and metalloproteinases, have been appointed as potential culprits [42]. Besides respiratory sequelae already described long-lasting symptoms that persist for more than 12 weeks after the start of acute symptoms include fatigue, joint and chest pain, headaches, cognitive blunting ("brain fog"); rashes, mental health conditions including depression and thromboembolic disease. Interestingly, some of these symptoms also occur in serotonin-related syndromes, such as carcinoid syndrome and psychiatric conditions [43]. Indeed, psychiatric disorders such as depression and anxiety [44], and neurocognitive conditions such as memory, attention, and concentration disorders are more frequent in patients with NEN associated with carcinoid syndrome [45]. Whether 5-HT is also involved in postCOVID-19 syndrome is unknown but understanding the roles of 5-HT and other neurotransmitters like GRP and CRGP is urgently needed.

Metformin, an oral antidiabetic drug that targets IR, was described as the first agent that reverses lung fibrosis in a bleomycin model of lung fibrosis in mice [46]. Acting on inflammation, metformin seems to reduce the risk of mortality in patients with COVID-19 and diabetes [47]. Although not demonstrated, it was hypothesized that somatostatin analogues could potentially reduce the incidence of carcinoid heart disease by preventing carcinoidrelated fibrosis associated with chronic high levels of 5-HT [20]. Additionally, tryptophan hydroxylase inhibition is a promising novel therapy for serotonin-induced fibrosis [48].

Serotonin and COVID-19 Severity in

Diabetes and Obesity
SARS-CoV-2 Infection as Part of a Syndemic: Final Remarks

Until now, the management of COVID-19 pandemic has been mostly driven by epidemiologists, internists, and intensivists. The fact that COVID-19 is more than an acute respiratory disease, points toward the fact that this disease should be considered a syndemic health condition, according to the anthropological concept that biological and social interactions are crucial for disease prognosis and should all be considered with managing and treating the condition as well as by health policies $[49,50]$. Syndemic criteria include: (1) two (or more) diseases or health conditions cluster within a specific population; (2) contextual and social factors create the conditions in which two (or more) diseases or health conditions cluster; and (3) the clustering of diseases results in adverse disease interaction, either biological or social or behavioral, increasing the health burden of affected populations [49]. If the context variable is introduced, it implies that from the anthropological point of view, public health policies should take into consideration the organic specificities that are ethnically/genetically differentiated and the interactions that result from the adaptation process to the social and demographic environment. In this case, the context works as an analytical tool that establishes methodological boundaries. Diabetes prevalence and population density are not uniform across the world, neither is average life expectancy, nor food availability and environmental conditions. This implies that the pandemic public health policies, overshadowed by the impact of COVID-19, will have distinctive impacts according to the different contexts.

So, the COVID-19 pandemic should be managed in a global context that integrates not only the chronic noncommunicable diseases, considered the 21 st century's epidemics driven by the human lifestyle modifications, but also the social, ethnic, and environmental conditions [51, 52]. In that sense, the SARS-CoV-2 infection pandemic can be considered the first global acute infectious disease caused by human-driven environment aggressions, which in turn has mainly affected the western lifestyle in developed countries. In sum, SARS-CoV-2 threat could also be an opportunity for a global change into the world as it was previously known.

\section{Conflict of Interest Statement}

The authors have no conflicts of interest to declare.

Neuroendocrinology 2022;112:1039-1045 DOI: $10.1159 / 000522115$ 


\section{Funding Sources}

No funding was received for this study.

\section{Author Contributions}

Paper conception and design: A.P.S. Manuscript elaboration: A.P.S., C.F.C., and S.S.P. Manuscript revision: M.P.M. Final approval of the version to be published: A.P.S., C.F.C., S.S.P., and M.P.M. Agreement to be accountable for integrity of the study: A.P.S., C.F.C., S.S.P., and M.P.M.

\section{References}

1 Veiga-Fernandes H, Mucida D. Neuro-immune interactions at barrier surfaces. Cell. 2016 May 5;165(4):801-11.

2 Godinho-Silva C, Cardoso F, Veiga-Fernandes $\mathrm{H}$. Neuro-immune cell units: a new paradigm in physiology. Annu Rev Immunol. 2019 Apr 26;37:19-46.

3 Ye Q, Wang B, Mao J. The pathogenesis and treatment of the "Cytokine Storm" in COVID-19. J Infect. 2020 Jun;80(6):607-13.

4 Chiappetta S, Sharma AM, Bottino V, Stier C. COVID-19 and the role of chronic inflammation in patients with obesity. Int J Obes. 2020; 44(8):1790-2.

5 Popkin BM, Du S, Green WD, Beck MA, Algaith $\mathrm{T}$, Herbst $\mathrm{CH}$, et al. Individuals with obesity and COVID-19: a global perspective on the epidemiology and biological relationships. Obes Rev. 2020 Nov;21(11):e13128.

6 Watanabe M, Risi R, Tuccinardi D, Baquero CJ, Manfrini S, Gnessi L. Obesity and SARSCoV-2: a population to safeguard. Diabetes Metab Res Rev. 2020 Apr 21:e3325.

7 Bode B, Garrett V, Messler J, McFarland R, Crowe J, Booth R, et al. Glycemic characteristics and clinical outcomes of COVID-19 patients hospitalized in the United States. J Diabetes Sci Technol. 2020 Jul;14(4):813-21.

8 Singh AK, Khunti K. Assessment of risk, severity, mortality, glycemic control and antidiabetic agents in patients with diabetes and COVID-19: a narrative review. Diabetes Res Clin Pract. 2020 Jul;165:108266.

9 Kruglikov IL, Scherer PE. The role of adipocytes and adipocyte-like cells in the severity of COVID-19 infections. Obesity. 2020 Jul; 28(7):1187-90.

10 Shoelson SE, Lee J, Goldfine AB. Inflammation and insulin resistance. J Clin Invest. 2006 Jul;116(7):1793-801.

11 Pearson-Stuttard J, Zhou B, Kontis V, Bentham J, Gunter MJ, Ezzati M, et al. Worldwide burden of cancer attributable to diabetes and high body-mass index: a comparative risk assessment. Lancet Diabetes Endocrinol. 2018 Jun;6(6):e6-e15.

12 Avgerinos KI, Spyrou N, Mantzoros CS, Dalamaga M. Obesity and cancer risk: Emerging biological mechanisms and perspectives. Metabolism. 2019 Mar;92:121-35.

13 Santos AP, Santos AC, Castro C, Raposo L, Pereira SS, Torres I, et al. Visceral obesity and metabolic syndrome are associated with well-differentiated gastroenteropancreatic neuroendocrine tumors. Cancers. 2018 Aug 27;10(9):293.
14 Berkovic MC, Cacev T, Ivkovic TC, ZjacicRotkvic V, Kapitanovic S. New insights into the role of chronic inflammation and cytokines in the etiopathogenesis of gastroenteropancreatic neuroendocrine tumors. Neuroendocrinology. 2014;99(2):75-84.

15 Pereira SS, Pereira R, Santos AP, Costa MM, Morais T, Sampaio P, et al. Higher IL-6 peritumoural expression is associated with gastro-intestinal neuroendocrine tumour progression. Pathology. 2019 Oct;51(6):593-9.

16 Dawson CA, Linehan JH, Rickaby DA, Bronikowski TA. Kinetics of serotonin uptake in the intact lung. Ann Biomed Eng. 1987;15(2): 217-27.

17 Kaltsas GA, Besser GM, Grossman AB. The diagnosis and medical management of advanced neuroendocrine tumors. Endocr Rev. 2004 Jun;25(3):458-511.

18 Torniai M, Scortichini L, Tronconi F, Rubini C, Morgese F, Rinaldi S, et al. Systemic treatment for lung carcinoids: from bench to bedside. Clin Transl Med. 2019 Jul 4;8(1):22.

19 Laskaratos FM, Rombouts K, Caplin M, Toumpanakis C, Thirlwell C, Mandair D. Neuroendocrine tumors and fibrosis: an unsolved mystery? Cancer. 2017 Dec 15;123(24): 4770-90.

20 Clement D, Ramage J, Srirajaskanthan R. Update on pathophysiology, treatment, and complications of carcinoid syndrome. J Oncol. 2020;2020:8341426.

21 Martin AM, Young RL, Leong L, Rogers GB, Spencer NJ, Jessup CF, et al. The diverse metabolic roles of peripheral serotonin. Endocrinology. 2017 May 1;158(5):1049-63.

$22 \mathrm{Ha}$ S, Jin B, Clemmensen B, Park P, Mahboob $\mathrm{S}$, Gladwill V, et al. Serotonin is elevated in COVID-19-associated diarrhoea. Gut. 2021 Jan 5;70(10):2015.

23 Marik PE, Iglesias J, Varon J, Kory P. A scoping review of the pathophysiology of COVID-19. Int J Immunopathol Pharmacol. 2021 Jan;35:20587384211048026.

24 Attademo L, Bernardini F. Are dopamine and serotonin involved in COVID-19 pathophysiology? Eur J Psychiatr. 2021;35(1):62.

25 Heath TP, Melichar JK, Nutt DJ, Donaldson LF. Human taste thresholds are modulated by serotonin and noradrenaline. J Neurosci. 2006 Dec 6;26(49):12664-71.

26 Gaudry Q. Serotonergic modulation of olfaction in rodents and insects. Yale J Biol Med. 2018 Mar;91(1):23-32.
27 Gautier JF, Ravussin Y. A new symptom of COVID-19: loss of taste and smell. Obesity. 2020 May;28(5):848.

28 Franchini M, Liumbruno GM, Pezzo M. COVID-19 vaccine-associated immune thrombosis and thrombocytopenia (VITT): diagnostic and therapeutic recommendations for a new syndrome. Eur J Haematol. 2021 May 13;107(2): 173 .

29 Tardy B, Lecompte T, Mullier F, Vayne C, Pouplard C. Detection of platelet-activating antibodies associated with heparin-induced thrombocytopenia. J Clin Med. 2020 Apr 24; 9(4): 1226.

30 Schoenichen C, Bode C, Duerschmied D. Role of platelet serotonin in innate immune cell recruitment. Front Biosci. 2019 Jan 1;24:51426.

31 Hoertel N, Sánchez-Rico M, Vernet R, Beeker $\mathrm{N}$, Jannot AS, Neuraz A, et al. Association between antidepressant use and reduced risk of intubation or death in hospitalized patients with COVID-19: results from an observational study. Mol Psychiatr. 2021;26(9):5199-212.

32 Lenze EJ, Mattar C, Zorumski CF, Stevens A, Schweiger J, Nicol GE, et al. Fluvoxamine versus placebo and clinical deterioration in outpatients with symptomatic COVID-19: a randomized clinical trial. JAMA. 2020 Dec 8; 324(22):2292-300.

33 Seftel D, Boulware DR. Prospective cohort of fluvoxamine for early treatment of coronavirus disease 19. Open Forum Infect Dis. 2021; 8(2):ofab050.

34 Jalali F, Rezaie S, Rola P, Kyle-Sidell C. COVID-19 pathophysiology: are platelets and serotonin hiding in plain sight? Neuroendocrinol. 2021.

35 Zimering MB, Razzaki T, Tsang T, Shin JJ. Inverse association between serotonin 2 a receptor antagonist medication use and mortality in severe COVID-19 infection. Endocrinol Diabetes Metab J. 2020;4(4):1-5.

36 Rossi G, Cavazza A, Spagnolo P, Sverzellati N, Longo L, Jukna A, et al. Diffuse idiopathic pulmonary neuroendocrine cell hyperplasia syndrome. Eur Respir J. 2016 Jun;47(6):1829_ 41.

37 Jin L, Wang Z, Qi X. Diffuse idiopathic pulmonary neuroendocrine cell hyperplasia: Case series and a review of the literature. Medicine. 2018 Dec;97(52):e13806.

38 Pan Y, Guan H. Imaging changes in patients with 2019-nCov. Eur Radiol. 2020 Jul;30(7): 3612-3. 
39 Mahase E. Covid-19: what do we know about "long covid"? Br Med J. 2020;370.

40 Nalbandian A, Sehgal K, Gupta A, Madhavan MV, McGroder C, Stevens JS, et al. Post-acute COVID-19 syndrome. Nat Med. 2021 Apr; 27(4):601-15

41 George PM, Wells AU, Jenkins RG. Pulmonary fibrosis and COVID-19: the potential role for antifibrotic therapy. Lancet Respir Med. 2020 Aug;8(8):807-15.

42 Ottenhoff MC, Ramos LL, Potters W, Janssen ML, Hubers D, Hu S, et al. Predicting mortality of individual COVID-19 patients: a multicenter Dutch cohort. BMJ Open. 2021 Jul 19; 11(7): 047347.

43 Del Rio C, Collins LF, Malani P. Long-term health consequences of COVID-19. JAMA. 2020 Oct 5;324(17):1723.
44 Lewis AR, Wang X, Magdalani L, D’Arienzo P, Bashir C, Mansoor W, et al. Health-related quality of life, anxiety, depression and impulsivity in patients with advanced gastroenteropancreatic neuroendocrine tumours. World J Gastroenterol. 2018 Feb 14;24(6):671-9.

45 Chambers AJ, Longman RS, Pasieka JL, Dixon E, Rorstad O, Rach-Longman K, et al. Impairment of cognitive function reported by patients suffering from carcinoid syndrome. World J Surg. 2010 Jun;34(6):1356-60.

46 Rangarajan S, Bone NB, Zmijewska AA, Jiang $S$, Park DW, Bernard K, et al. Metformin reverses established lung fibrosis in a bleomycin model. Nat Med. 2018 Aug;24(8):1121-7.

47 Crouse A, Grimes T, Li P, Might M, Ovalle F, Shalev A. Metformin use is associated with reduced mortality in a diverse population with Covid-19 and diabetes. medRxiv. 2020 Jul 31.
48 Bader M. Inhibition of serotonin synthesis: a novel therapeutic paradigm. Pharmacol Ther. 2020 Jan;205:107423.

49 Singer M, Bulled N, Ostrach B, Mendenhall E. Syndemics and the biosocial conception of health. Lancet. 2017 Mar 4;389(10072):94150 .

50 Irons R. Pandemic or syndemic? Re-framing COVID-19 disease burden and underlying health conditions. Soc Anthropol. 2020 May 19.

51 Horton R. Offline: 2019-nCoV outbreak-early lessons. Lancet. 2020 Feb 1;395(10221):322.

52 Hill MA, Sowers JR, Mantzoros CS. Commentary: COVID-19 and obesity pandemics converge into a syndemic requiring urgent and multidisciplinary action. Metabolism. 2021 Jan;114:154408. 